\title{
Inflationary Kaluza-Klien Cosmic String in Scalar-Tensor Theory of Gravitation
}

\author{
J. Satish*, R. Venkateswarlu** \\ * Department of BS\&H, Mathematics, Vignan's Institute of Engineering for women, India \\ ** GITAM School of Int'l Business, GITAM University, India
}

\begin{abstract}
Article Info
ABSTRACT

Article history:

Received Jun 14, 2014

Revised Aug 7, 2014

Accepted Aug 21, 2014

Keyword:

We have investigated a spatially homogeneous and anisotropic Kaluz-Klien space time with cosmic strings in the presence of Sen Dunn theory as source. Exact solutions of the Einstein field equations are presented via a suitable power law assumption. Some of models obey geometric strings, massive strings and Takabayasi's equation of state. We find that the constant value of deceleration parameter is reasonable for the present day universe. Some physical and geometric behaviour of the models are also discussed.
\end{abstract}

Anisotropy

Cosmology

Inflation

Scalar tensor theory

Strings

Copyright $@ 2014$ Institute of Advanced Engineering and Science. All rights reserved.

\section{Corresponding Author:}

\section{J. Satish,}

Dept of BS\&H, Mathematics,

Vignan's Institute of Engineering for women,

Visakhapatnam, 530046, India.

Email: mathssatish@gmail.com

\section{INTRODUCTION}

The history of cosmology has witnessed many conjectures. The latest is inflation in the very early universe meaning thereby an exponential accelerated cosmic expansion associated with the standard model. Inflation, apart from providing a solution to a number of problems faced by Fridermann models, is believed to have induced tiny in-homogeneities in the early universe, which evolved into galaxies and other cosmic structures. Various types of inflationary models are being considered, such as string cosmology. String theory is a powerful tool to describe the early phase of universe. In a string theory, we have a mode of describing the graviton. It makes strings more useful to all other cosmological structures. The classical theory of these strings was developed by Stachel [1]. Letelier [2] presented a model of string-dust in which incoherent matter particles are attached to geometric string along its extension. The presence of strings results into anisotropy in the space time. Strings are not observed in the present epoch. For a physically meaningful string model, it is desirable that either strings disappear at a certain epoch of cosmic evolution or it has a particle dominated future asymptote with undetectable strings [3].

The concept of string theory was developed to describe events at the early stages of the evolution of the universe. So strings are important in the early stages of evolution of the universe before the particle creation. The present day observations do not rule out the possible existence of large scale networks of string in the early universe. Gauge theories with spontaneous symmetry breaking in elementary particle physics have given rise to anopontensive study of cosmic strings. It appears that after big bang the universe may have experienced a number of phase transitions Linde [4]. These phase transitions can produce vacuum domain structures such as domain walls, string and monopoles Kibble [5], Zel'dovich [6] of all these cosmological structures, cosmic strings have excited perhaps the most interest. Cosmic strings may act as gravitational 
lenses Vilenkin [7], [8] and may give rise to density perturbations leading to formation of galaxies. Kibble [9] mentioned that the presence of strings in the early universe can be explained using grand unified theories. These are two major fields that the string theory ought to illuminate some day; particle physics and cosmology. Particle physics addresses the microscopic extreme while cosmology the microscopic extreme. Relativists and particle theorists have both identified the important problem of reconciling quantum theory with general relativity. The prospect of achieving this attracts both of them to string theory.

Modified theories of gravity have been the subject of study for the last few decades. As an alternative to Einstein's theory of gravitation, Sen and Dunn [10] have proposed a new scalar-tensor theory of gravitation in which both the scalar and tensor fields have intrinsic geometrical significance. The scalar field in this theory is characterized by the function $\phi=\phi\left(x^{i}\right)$ where $x^{i}$ are coordinates in the four dimensional Lyra manifold and the tensor field is identified with the metric tensor $g_{i j}$ of the manifold. The field equations given by Sen and Dunn for the combined scalar and tensor fields are:

$$
R_{i j}-\frac{1}{2} g_{i j} R=\omega \phi^{-2}\left(\phi_{, i} \phi_{, j}-\frac{1}{2} g_{i j} \phi_{, k} \phi^{, k}\right)-\phi^{-2} T_{i j}
$$

Where $\omega=\frac{3}{2}, R_{i j}$ and $R$ are respectively the usual Ricci-tensor and Riemaan-curvature scalar (in our units $C=8 \pi G=1)$.

Recently, considerable interest have been evinced in theories of more than four dimensions, in which the extra dimensions are compacted to small size in the course of evolution of the universe [11]. The cosmological study in higher dimensional space time are necessitated, even made urgent, by the growing belief that the nature of space time in the universe are higher than four. Chatterjee [12] studied massive strings in higher dimensional homogeneous space time. Krori et al. [13] discussed Bianchi type-1 higher dimensional cosmologies and concluded that, physically, strings will be like geometric string, and matter and strings coexist throughout the evolution of the universe. They mentioned that cosmic strings with some specific orientation do not occur in Bianchi type-V cosmology. Rahaman et al. [14] discussed some string cosmological models in a higher dimensional spherically symmetric space time based on Lyra's geometry. Venkateswarlu [15] constructed higher dimensional string cosmological models in scale covariant theory of gravitation. Recently Mohanty et al. [16] and Mohanty and Mahanta [17] constructed various higher dimensional string cosmological models and studied their geometrical and physical behaviors. Dunn [18], Halford [19], Singh [20], Reddy [21], [22], Roy and Chatterjee [23], [24], Reddy and Venkateswarlu [25], and Mukherjee [26], [27] are some of the authors who have studied various aspects of this scalar-tensor theory of gravitation. Very recently Venkateswarlu, Satish and Pavan Kumar [28] have studied the Bianchi Type -I cosmic strings in this theory.

In this paper, we made an attempt to solve the five dimensional spherically symmetric space-time in the context of cosmic strings in a new scalar-tensor theory of gravitation proposed by Sen and Dunn [10]. Exact solutions of the field equations are obtained viz. power law type assumption.

\section{METRIC AND FIELD EQUATIONS}

We consider the line element for the five-dimensional Kaluza-klien model is:

$$
d s^{2}=d t^{2}-e^{A(t)}\left\lfloor d r^{2}+r^{2} d \theta^{2}+r^{2} \sin ^{2} \theta d \phi^{2}\right\rfloor-e^{B(t)} d y^{2}
$$

Where A and B are functions of cosmic time t only. The fifth coordinate $y$ is also assumed to be space like coordinate.

The energy momentum tensor for a cloud of massive strings that can be written as:

$$
\mathrm{T}_{i j}=\rho u_{i} u_{j}-\lambda x_{i} x_{j}
$$

Here $\rho$ is the rest energy density of the cloud of strings with particles attached to them, $\lambda$ is the tension density of the strings and $\rho=\rho_{p}+\lambda, \rho_{p}$ being the energy density of the particles. From all the three energy conditions (weak, strong and dominant) for string model, one can find that $\rho>0$ and $\rho_{p} \geq 0$ 
and the sign of $\lambda$ is unrestricted. One can note that the field equations with $\lambda<0$ can be interpreted as the presence of anisotropic fluid with pressure different from zero along the direction of the string. The velocity $u^{i}$ describes the five - velocity which has components $(1,0,0,0,0)$ for a cloud of particles and $x^{i}$ represents the direction of string which will satisfy:

$$
u^{i} u_{i}=-x^{i} x_{i}=1 \text { and } u^{i} x_{i}=0
$$

The direction of the strings is taken to be along $X_{4}-$ axis so that we have $X^{i}=\left(0,0,0,0, e^{-B}\right)$. Now the field equations for the metric (2) are given by:

$$
\begin{aligned}
& \ddot{A}+\frac{3}{4} \dot{A}^{2}+\frac{\ddot{B}}{2}+\frac{1}{4} \dot{B}^{2}+\frac{1}{2} \dot{A} \dot{B}=\frac{\omega}{2}\left(\frac{\dot{\phi}}{\phi}\right)^{2} \\
& \frac{3}{2} \ddot{A}+\frac{3}{2} \dot{A}^{2}=\frac{\omega}{2}\left(\frac{\dot{\phi}}{\phi}\right)^{2}+\frac{\lambda}{\phi^{2}} \\
& \frac{3}{4} \dot{A}^{2}+\frac{3}{4} \dot{A} \dot{B}=\frac{\rho}{\phi^{2}}-\frac{\omega}{2}\left(\frac{\dot{\phi}}{\phi}\right)^{2}
\end{aligned}
$$

Where the overhead dot denotes ordinary differentiation with respect to $t$.

\section{SOLUTIONS TO THE FIELD EQUATIONS}

The field Equation (5)-(7) are a system of three equations with five unknown parameters A, B, $\phi, \rho$ and $\lambda$. We need two additional conditions to get a deterministic solution of the above system of equations. Thus we present the solutions of the field equations in the following physically meaningful cases .To get a realistic solution here we assume that:

$$
e^{A}=t^{m n} \quad \text { and } \quad e^{B}=t^{n}
$$

Where $m$ and $n$ are arbitrary constants. Then from Equation (5) we obtain:

$$
\phi=\phi_{0} t^{k}
$$

Where $\phi_{0}$ is an arbitrary constant and $k=\left(\frac{n^{2}\left(3 m^{2}+2 m+1\right)-2 n(2 m+1)}{2 \omega}\right)^{\frac{1}{2}}$.

Thus the five dimensional Kaluza-klien space-time is:

$$
d s^{2}=d t^{2}-t^{m n}\left[d r^{2}+r^{2} d \theta^{2}+r^{2} \sin ^{2} \theta d \phi^{2}\right]-t^{n} d y^{2}
$$

The string energy density $\rho$, tension density $\lambda$, the particle density $\rho_{p}$, the scalar expansion $\theta$, the shear scalar $\sigma$, spatial volume $V$ and the deceleration parameter $q$ are given by:

$$
\begin{aligned}
& \rho=\phi_{0}^{2}\left(\frac{n^{2}\left(6 m^{2}+5 m+1\right)-2 n(2 m+1)}{4 t^{2-2 k}}\right) \\
& \lambda=\phi_{0}^{2}\left(\frac{n^{2}\left(3 m^{2}-2 m-1\right)-2 n(2 m+1)}{4 t^{2-2 k}}\right)
\end{aligned}
$$




$$
\begin{aligned}
& \rho_{p}=\rho-\lambda=\left(\frac{n^{2}\left(3 m^{2}+7 m+2\right)-2 n(2 m+2)}{4 t^{2-2 k}}\right) . \\
& \theta=\frac{(3 m+1) n}{2 t} \\
& \sigma=\frac{n \sqrt{117 m^{2}+24 m+7}}{6 t} \\
& V=t^{(3 m n+n)} \\
& q=\frac{(3-n-3 m n)}{(3 m n+n)}
\end{aligned}
$$

The energy conditions viz., $\rho>0, \lambda>0$ and $\rho_{p}>0$ are identically satisfied for all $m>0$ and $n>0$. Since $\frac{\sigma}{\theta}=$ constant, the model given by Equation (10) does not approach isotropy at any stage. The spatial volume of the model increases with the increase in time. The model given by equation (10) for a cloud of cosmic strings possess a line singularity as $\rho, \lambda, \theta$ and $\sigma$ tend to infinity and spatial volume tend to zero at initial epoch $t=0$.

Case 3.1: The case $\rho=\lambda$ refers to geometric strings. If $m=-2$, the solution of the field Equations (5)-(7) is given by:

$$
e^{A}=t^{-2 n}, \quad e^{B}=t^{n}
$$

And the scalar field:

$$
\phi=\phi_{1} t^{k_{1}}
$$

Where $\phi_{1}$ is an arbitrary constant and $k_{1}=\left(\frac{n(9 n+6)}{2 \omega}\right)^{\frac{1}{2}}, n<-\frac{2}{3}$.

The Kailza-Klien five dimensional model in this case reduces to the form:

$$
d s^{2}=d t^{2}-t^{-2 n}\left[d r^{2}+r^{2} d \theta^{2}+r^{2} \sin ^{2} \theta d \phi^{2}\right]-t^{n} d y^{2}
$$

The string energy density $\rho$ and tension density $\lambda$ are:

$$
\lambda=\rho=\phi_{1}^{2} \frac{\left(15 n^{2}+6 n\right)}{t^{2-2 k_{1}}} .
$$

The particle density $\rho_{p}$, and the deceleration parameter $q$ are given by:

$$
\begin{aligned}
& \rho_{p}=\rho-\lambda=0, \\
& q=-\left(1+\frac{3}{5 n}\right) .
\end{aligned}
$$

It is observed that the special volume $\mathrm{V}$ is zero at $t=t_{0}$ where $t_{0}=0$ and expansion scalar is infinite, which shows that the universe starts evolving with zero volume at $t=t_{0}$ with an infinite rate of expansion. The model has a "Barrel type" singularity at initial epoch for $n<-\frac{2}{3}$. The string energy density 
$\rho$ and tension density $\lambda$ and the shear scalar $\sigma$ diverge at the initial singularity. As t increases, the scale factors and spatial volume increases but the expansion scalar decreases. Thus, the rate of expansion slows down with increase in time. Also the scalar expansion $\theta$, the shear scalar $\sigma$ decreases as $\mathrm{t}$ increases. As $t \rightarrow \infty$ volume $\mathrm{V}$ becomes infinite whereas $\rho, \lambda, \theta$ and $\sigma$ tend to zero. Therefore, the model would essentially give an empty universe for large time $\mathrm{t}$ and it has 'Cigar type' singularity at late times provided for $n<-\frac{2}{3}$. For expanding universe, the scale factor $\mathrm{A}(\mathrm{t})$ should be an increasing function of time while the scale factor $\mathrm{B}(\mathrm{t})$ corresponding to extra dimension should decrease or be a constant to have comactification of extra dimension. Thus the extra dimension $\mathrm{B}(\mathrm{t})$ contracts whereas $\mathrm{A}(\mathrm{t})$ expands indefinitely with time for $n<0$.

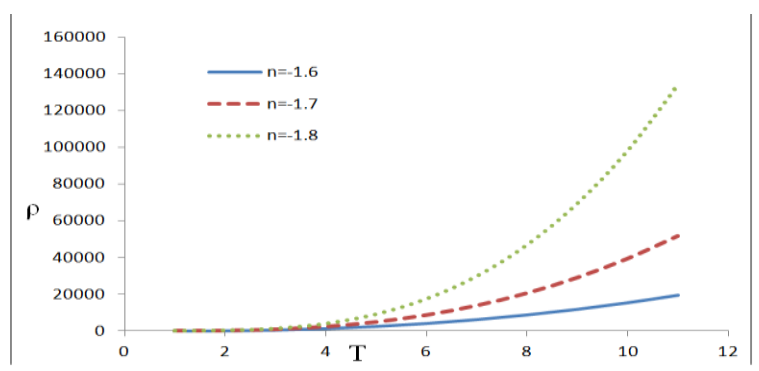

Figure 1. The plot of string energy density $\rho$ vs. $t$ for $n=-1.6, n=-1.7, n=-1.8$ and $\omega=1.5$

From Equation (21), we observed that the rest density $\rho$ is increasing function of time and always positive. We have plotted the graphs of energy density versus time in figure-1. It is clear that the rest energy density remains positive.

Case 3.2: Now we consider the massive string, $(\rho+\lambda=0)$ i.e., the sum of rest energy density and tension density for cloud of strings vanish. Then we have $m=o$. the solution of the field Equation (5)-(7) can be expressed as:

$$
e^{A}=1 \text { and } \quad e^{B}=t^{n}
$$

And the scalar field is given by:

$$
\phi=\phi_{2} t^{k_{2}}
$$

Where $\phi_{2}$ is an arbitrary constant and $k_{2}=\left(\frac{n^{2}-2 n}{2 \omega}\right)^{\frac{1}{2}}$

The model, for massive strings, reduces to:

$$
d s^{2}=d t^{2}-\left[d r^{2}+r^{2} d \theta^{2}+r^{2} \sin ^{2} \theta d \phi^{2}\right]-t^{n} d y^{2}
$$

The string energy density $\rho$ and tension density $\lambda$ are:

$$
\rho=-\lambda=\phi_{2}^{2} \frac{\left(n^{2}-2 n\right)}{t^{2-2 k_{2}}}
$$

The particle density is:

$$
\rho_{p}=2 \phi_{2}^{2} \frac{\left(n^{2}-2 n\right)}{t^{2-2 k_{2}}}
$$


If $n=2, \lambda=\rho=0$ this shows that the cosmic strings do not exist. It is observed that the scalar field becomes a constant when $n=2$. This situation lead to the general relativity case. And the kinematical parameters are given by:

$$
\begin{aligned}
& \text { Scalar expansion } \theta=\frac{n}{2 t}, \\
& \text { Shear scalar } \sigma=\frac{\sqrt{7} n}{6 t}, \\
& \text { Spatial volume } V=\sqrt{g}=t^{n}, \\
& \text { Deceleration parameter } q=\frac{-a \ddot{a}}{\dot{a}^{2}}=\frac{1}{n}-1 .
\end{aligned}
$$

The dominant energy conditions implies that $\rho>0$ and $\rho^{2} \geq \lambda^{2}$. These energy conditions do not restrict the sign of $\lambda$, accordingly the expressions given by equation (26) satisfies all these conditions. We observe that $\frac{\rho_{p}}{|\lambda|}=2$. Since $\frac{\rho_{p}}{|\lambda|}>1$, thus we may conclude that the particles dominate over the strings in this model.

It is easy to see that $\frac{\sigma}{\theta}=.88$, for our models. The present upper limit of $\frac{\sigma}{\theta}$ is $10^{-5}$ obtained from indirect arguments concerning the isotropy of the primordial blackbody radiation [29]. The $\frac{\sigma}{\theta}$ for our models is considerably greater than the present value. This fact indicates that our solutions represent the early stages of evolution of the universe.

As time increases the scale factors B (t) increase indefinitely indicating that there is no compactification of extra dimension with time in the model for massive strings. Since $\frac{\sigma}{\theta}=$ Constant, the anisotropy is maintained throughout and the model inflates since $q<0$ for all ' $n>1$ '. The present day Universe is undergoing accelerated expansion. It may be noted that the current observations of SNe Ia and the CMBR favour accelerating models $(q<0)$.

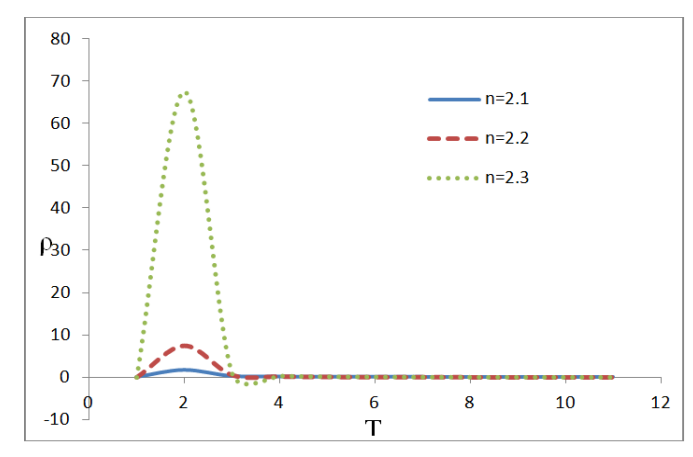

Figure 2. The plot of string energy density $\rho$ vs. $t$ for $n=2.1, n=2.2, n=2.3$ and $\omega=1.5$

From Equation (26), it is observed that the rest energy density $\rho$ is a constant function of time and always $\rho>0$. This is shown in Figure 2. This means there is no density evolution in this set up. A possible reason for no evolution of density is that expansion of the Universe could be much rapid in which matter do not get time to re-adjust on expansion range or may be other unknown dominated effect which not incorporated in potential functions of this space-time for power-law.

Case3.3: The P-strings or Takabayasi strings are represented by $\rho=(1+\xi) \lambda, \xi>0$. The solution of the field equations (5)-(7) is given by: 


$$
e^{A}=t^{\left(\frac{2+\xi}{\xi-1}\right) n} \text { and } e^{B}=t^{n}
$$

And the scalar field is given by:

$$
\phi=\phi_{3} t^{k_{3}}
$$

Where $\phi_{3}$ is an arbitrary constant $k_{3}=\left(\frac{n^{2}\left(4 \xi^{2}+4 \xi+1\right)-2 n\left(3 \xi^{2}-3\right)}{2 \omega(\xi-1)^{2}}\right)^{\frac{1}{2}}$.

The line element for P-strings reduces to the form:

$$
d s^{2}=d t^{2}-t^{2 n\left(\frac{2+\xi}{\xi-1}\right)}\left[d r^{2}+r^{2} d \theta^{2}+r^{2} \sin ^{2} \theta d \phi^{2}\right]-t^{2 n} d y^{2}
$$

Now the string energy density $\rho$, tension density $\lambda$, and the particle density $\rho_{p}$ are given by:

$$
\rho=(1+\xi) \lambda=3 n\left[\frac{n(5+4 \xi)-2(\xi-1)}{(\xi-1)^{2} 4 t^{2-2 k_{3}}}\right]
$$

And the kinematical parameters are given by:

Scalar expansion $\theta=\frac{n(5+4 \xi)}{2 t}$,

Shear scalar $\sigma=\frac{n}{6 t(\xi-1)} \sqrt{148 \xi^{2}+458 \xi+427}$,

Spatial volume $V=\sqrt{g}=t^{\frac{n(4 \xi+5)}{(\xi-1)}}$,

Deceleration parameter $q=\frac{-a \ddot{a}}{\dot{a}^{2}}=\frac{\xi(3-4 n)-(3+5 n)}{3 n(2 \xi+1)}$.

From the above parameters we observe that $\rho, \lambda$, the scalar expansion $\theta$, as well as the shear scalar $\sigma$ all decreases as the increase in time. At the singularity stage $t \rightarrow 0, v^{3} \rightarrow 0$ and $\rho, \lambda, \theta$, and $\sigma$ all infinitely large but at $t \rightarrow \infty, v^{3} \rightarrow \infty$ and $\rho, \lambda, \theta$, and $\sigma$ all vanish which represent an empty universe. But all these parameters remain finite and physically significant for all $t>0$. Hence we see that space-time admits a big-bang singularity but the rate of expansion of the universe decreases with increase of time. As time increases the scale factors $B$ and $A$ increase indefinitely indicating that there is no compactification of extra dimension with time.

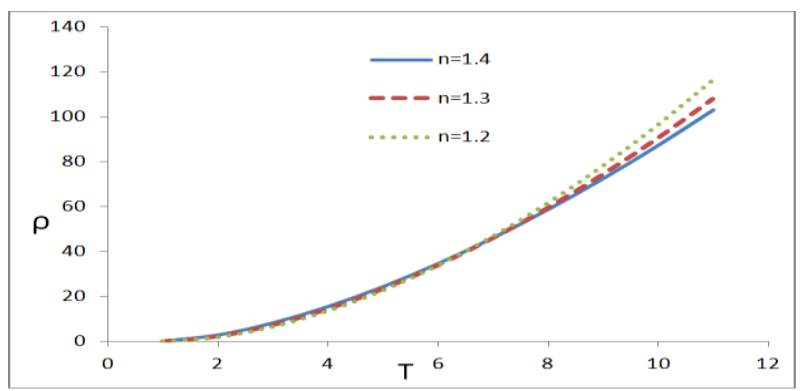

Figure 3. The plot of string energy density $\rho$ vs. $t$ for $n=1.2, n=1.3, n=1.4, \xi=4$ and $\omega=1.5$ 
From Equation (32), it is observed that the rest energy density $\rho$ is an increasing function of time and $\rho>0$ always. The rest energy density has been plotted versus time in Figure 3 . It is evident that the rest energy density remains positive in the evolution of universe.

Case 3.4: The case $\lambda=0$ corresponds to a dust filled universe without strings whose future asymptote is the Einstein -desitter universe. If $m=1$, the solution of the field Equation (5)-(7) is given by:

$$
e^{A}=e^{B}=t^{n}
$$

And the scalar field is given by:

$$
\phi=\phi_{4} t^{k_{4}}
$$

Where $\phi_{4}$ is an arbitrary constant and $k_{4}=\left(\frac{6 n(n-1)}{\omega}\right)^{\frac{1}{2}}$.

The line element in this case can be written as:

$$
d s^{2}=d t^{2}-t^{n}\left[d r^{2}+r^{2} d \theta^{2}+r^{2} \sin ^{2} \theta d \phi^{2}\right]-t^{n} d y^{2}
$$

The string energy density $\rho$ is:

$$
\rho=\phi_{4}^{2} \frac{\left(12 n^{2}-6 n\right)}{t^{2-2 k_{4}}}
$$

If $n=0$ or $1, \lambda=\rho=0$ this shows that the cosmic strings do not exist. It is observed that the scalar field becomes a constant in both cases i.e. when $n=0$, or $n=1$. This situation lead to the general relativity case. The particle density $\rho_{p}$, the scalar expansion $\theta$, the shear scalar $\sigma$, spatial volume $V$ and the deceleration parameter $q$ are given by:

$$
\begin{aligned}
& \rho_{p}=\rho-\lambda=\frac{\left(12 n^{2}-6 n\right)}{t^{2-2 k_{4}}} \\
& \theta=\frac{2 n}{t} \\
& \sigma=\frac{\sqrt{148} n}{6 t} \\
& V=t^{4 n} \\
& q=-\left(\frac{4 n(4 n-3)}{16 n^{2}}\right)
\end{aligned}
$$

Usually the model decelerate when $q>0$ and inflates when $q<0$. Here for $0<\mathrm{n}<\frac{3}{4}$, the model decelerate and inflates if $\mathrm{n}>\frac{3}{4}$ at any stage. Determination of the deceleration parameter from the count magnitude relation for galaxies is a difficult task due to evolutionary effects. The present value $q$ of the deceleration parameter obtained from observations [30] are $-1.27 \leq \mathrm{q} \leq 2$. Studies of galaxy counts from redshift surveys provide a value of $q=0.1$, with an upper limit of $q \leq 0.75$ [30]. Recent observations by Perlmutter et al [31], [32] and Riess et al [33] show that the deceleration parameter of the Universe is in the range $-1 \leq \mathrm{q} \leq 0$ and the present day Universe is undergoing accelerated expansion. It may be noted that though the current observations of SNe Ia and the CMBR favour accelerating models $(q<0)$, they do not altogether rule out the existence of the decelerating phase in the early history of our Universe which are also consistent with these observations [34]. 


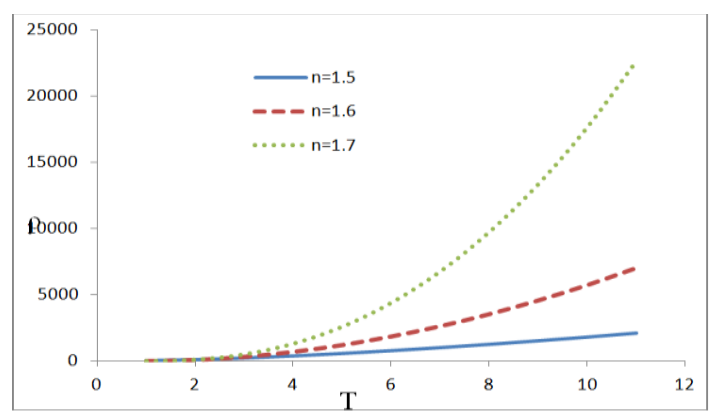

Figure 4. The plot of string energy density $\rho$ vs. $t$ for $n=1.5, n=1.6, n=1.7$ and $\omega=1.5$

From Equation (37), it is observed that the rest energy density $\rho$ is a constant function of time and $\rho>0$ always. The rest energy density has been graphed versus time in Figure 4. This means there is no density evolution in this set up. A possible reason for no evolution of density is that expansion of the Universe could be much rapid in which matter do not get time to re-adjust on expansion range or may be other unknown dominated effect which not incorporated in potential functions of this space-time for powerlaw.

\section{CONCLUSION}

We obtained the field equations of Sen - Dunn theory of gravitation with the help of five dimensional spherically symmetric metric in the context of cosmic strings. The solutions of the field are discussed in various physically meaningful cases namely, (i) geometric strings (i.e., $\lambda=\rho$ ), (ii) massive strings (i.e., $\lambda+\rho=0$ ), (iii) Takabayasi (or p-strings) strings i.e., $\rho=(1+\xi) \lambda$ and (iv) dust model (i.e., $\lambda=0$ ). It is observed that the power index of $n$ of the metric potential has a range of values for which the scalar field is real. Since $n$ has a specific range of values it is noticed that the extra dimension is amenable for reduction. Our model is in accelerating phase which is consistent with the recent observations. Thus the model (36) represents a realistic model. Inflationary (accelerated) universe scenario is important because it can solve some of the outstanding problems of standard 'big bang' cosmology. Therefore the study of cosmological models in the Sen-Dunn theory may be relevant for inflationary models. The classical scalar fields are essential in the study of the present day cosmological models. In view of the fact that there is an increasing intersection of these models, in recent years, scalar fields in general relativity and alternative theories of gravitation in the context of an inflationary Universe help us to describe the early stages of evolution of the Universe. Thus we may conclude that the scalar field in Sen-Dunn theory plays a significant role for the reduction of extra dimension in the context of cosmic strings.

\section{REFERENCES}

[1] Stachel J., Phys. Rev, 1980, Vol. 21, pp. 2171.

[2] Letelier P. S., Phys. Rev, 1983, Vol. 28, pp. 2414.

[3] Pant D .N., et al., Pramana J. Phys., 2003, Vol. 60, pp. 433.

[4] Linde A. D., Reports on Progess in Physics, 1979, Vol. 42, pp. 389.

[5] Kibble T. W. B., Physics Reports, 1980, Vol. 67, pp. 183.

[6] Zel'dovich Y. B., "Monthly Notices of the Royal astro- nomical Society," 1980, Vol. 192, pp. 663.

[7] Vilenkin A., Physical Review Letters, 1981, Vol. 46, pp. 1169.

[8] Vilenkin A., Physical Review, 1981, Vol. 28, pp. 852.

[9] Kibble T. W. B., Journal of Physics A, 1976, Vol. 9, pp. 1387.

[10] Sen D. K., et al., J. Math. Phys., 1971, Vol. 12, pp. 578.

[11] Salam A. Strathdee., J.Ann. Phys., 1982, Vol. 141, pp. 316.

[12] Chatterjee S., Gen. Rel. Grav., 1993, Vol. 25, pp. 1079.

[13] Krori K. D., et al., Gen. Rel. Grav., 1994, Vol. 26, pp. 265.

[14] Rahaman F., et al., J. Ind. J. Phys., 2002, Vol. 76B, pp. 747.

[15] Venkateswarlu R., et al., K. Astrophys. Space Sci., 2005, Vol. 298, pp. 403.

[16] Mohanty G., et al., Astrophys. Space Sci., 2007, Vol. 312, pp. 321.

[17] Mohanty G., et al., Astrophys. Space Sci., 2007, Vol. 312, pp. 301.

[18] Dunn K. A., J.Math. Phys., 1972, Vol. 15, pp. 2229

[19] Halford W. D., J.Math. Phys., 1972, Vol. 13, pp. 1699.

[20] Singh T., J. Math. Phys., 1975, Vol. 16, pp. 2109.

[21] Reddy D. R. K., J. Phys. A: Math. Nucl. Gen. 6, 1867. 
[22] Reddy D. R. K., J.Math. Phys., 1979, Vol. 20, pp. 23.

[23] Roy A. R., et al., Acta Phys. Hung. 1980, Vol. 48, pp. 383.

[24] Roy A. R., et al., Indian J. Pure Appl. Math. 1981, Vol. 12, pp. 659.

[25] Reddy D. R. K., et al., Astrophys. Space Sci., 1987, Vol. 135, pp. 287.

[26] Mukherjee B., Indian J. Pure Appl. Math., 2003, Vol. 34, pp. 121.

[27] Mukherjee B., Int. Journal of Academic Research, 2009, Vol. 1, pp. 18.

[28] Venkateswarlu, et al., "Ele," J. Theor. Phys., 2011, Vol. 8, pp. 25.

[29] Collins C. B., et al., Gen. Rel. Grav., 1980, Vol. 12, pp. 805.

[30] Schuecker R., et al., Astrophys. J. 1998, Vol. 496, pp. 635.

[31] Perlmutter S., et al., Nature, 1998, Vol. 319, pp. 51.

[32] Perlmutter S., et al., Astrophys. J., 1999, Vol. 517, pp. 565.

[33] Riess, et al., Astrophys. J. 1998, Vol. 116, pp. 1009.

[34] Vishwakarma R. G., Mon. Not. R. Soc, 2004, Vol. 345, pp. 545. 